PHYSICAL REVIEW FLUIDS 1, 050501 (2016)

\title{
Drop floating on a granular raft
}

\author{
Etienne Jambon-Puillet ${ }^{*}$ and Suzie Protière \\ UPMC, Sorbonne Universités, CNRS, UMR No. 7190, Institut Jean Le Rond d'Alembert, \\ F-75005 Paris, France
}

(Received 4 August 2016; published 12 September 2016)

\begin{abstract}
This paper is associated with a poster winner of a 2015 APS/DFD Milton van Dyke Award for work presented at the DFD Gallery of Fluid Motion. The original poster is available from the Gallery of Fluid Motion, http://dx.doi.org/10.1103/APS.DFD.2015.GFM.P0008
\end{abstract}

DOI: 10.1103/PhysRevFluids.1.050501

We study the interactions of a water drop with a monolayer of large and dense particles at an oil-water interface called a granular raft. If the particles are hydrophobic, we find that a water drop deposited on the raft will never coalesce with the water bath below. The raft prevents coalescence through particle bridging and the water drop will float indefinitely. When we increase the drop volume, its weight deforms the granular raft, which in turn deforms the drop. Finally, when we decrease the drop volume the contact line gets pinned and the raft wrinkles like an elastic solid.

When a droplet is gently deposited on a bath of the same liquid, the drop will merge almost immediately with the bath as the intermediate air film is rapidly evacuated when the drop's weight presses on it. This so-called coalescence process arises in many situations such as printing, the formation of clouds, or emulsions and foams where it reduces their stability and must be prevented. Coalescence can be delayed by adding surfactants in the liquid [1]. It can even be totally inhibited by preventing the contact of the liquid bath with the drop, either by renewing constantly the intermediate air film (when a flow is induced or when the liquid bath vibrates [2]) or by coating both interfaces with colloidal particles to provide steric repulsion [3]. Here, using granular rafts to protect the drop from the bath, we describe a simple system that completely suppresses coalescence and use the resulting floating drop to probe the mechanical response of the raft.

We sprinkle large $(d>0.2 \mathrm{~mm})$ and dense $\left(\rho_{p}>2.5 \mathrm{~g} \mathrm{~cm}^{-3}\right)$ particles at an oil-water interface, which spontaneously aggregate via capillary interactions into a compact monolayer that we call a
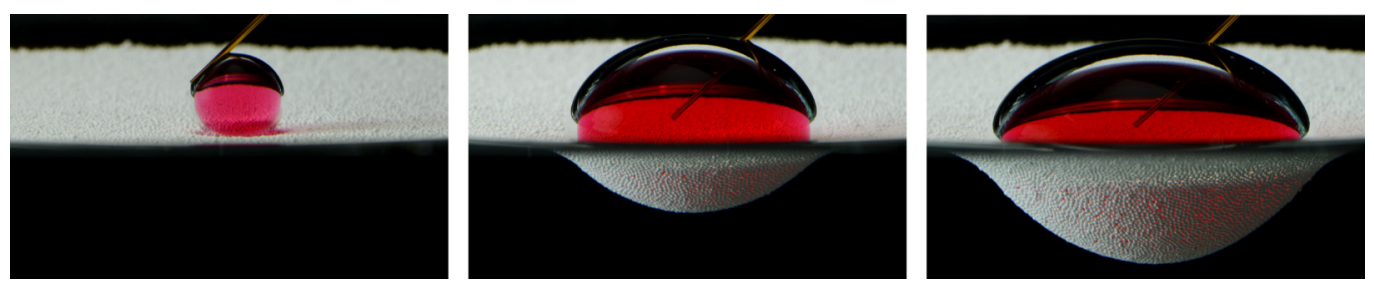

FIG. 1. Pictures of a water drop (dyed red) on a granular raft made of zirconium oxide particles at an oil-water interface (mean particle size $d=0.25 \mathrm{~mm}$, density $\rho_{p}=3.8 \mathrm{~g} \mathrm{~cm}^{-3}$, and contact angle $\theta_{y}=140^{\circ}$ ). The drop volume increases from left to right: $V=0.1,1.0$, and $2.0 \mathrm{~mL}$. DOI: http://dx.doi.org/10.1103/APS.DFD.2015.GFM.P0008

*Corresponding author: etienne.jambon-puillet@dalembert.upmc.fr

Published by the American Physical Society under the terms of the Creative Commons Attribution 3.0 License. Further distribution of this work must maintain attribution to the author(s) and the published article's title, journal citation, and DOI. 
ETIENNE JAMBON-PUILLET AND SUZIE PROTIÈRE

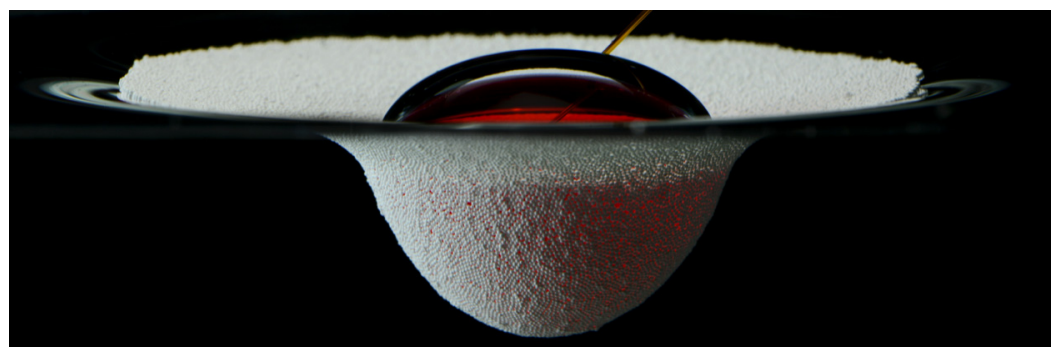

FIG. 2. The drop reaches its maximum size $V=3.2 \mathrm{~mL}$. DOI: http://dx.doi.org/10.1103/ APS.DFD.2015.GFM.P0008

granular raft [4]. Using a syringe needle, we then place a small water drop on the raft. If the particles are hydrophobic, coalescence with the water bath is inhibited indefinitely (Fig. 1, left, the water drop is dyed red). Indeed, a complex interface is formed: Particles are wetted by the water bath below as well as the water drop above and thus a stable oil layer is trapped in between the drop and the bath. This phenomenon is called particle bridging and can be observed in Pickering emulsions [5]. Here we present a macroscopic observation of this phenomenon.

When the drop volume is slowly increased by continuously injecting water with a capillary tube (Fig. 1, middle and right), the drop's weight pushes on the raft and induces very large deformations of the interface. At large volumes, when gravity dominates surface tension, the drop adopts an unusual shape: Its lower portion in contact with the raft is prolate and deeply immersed in water while its bare upper surface is oblate (Fig. 2). We can also incrementally decrease the drop volume by withdrawing water with the same capillary tube. In this case the drop does not go back to its original shape: The contact line stays pinned and the portion of the raft in contact with the drop is put under compression and starts to buckle. Finally, wrinkles appear in the azimuthal direction and grow in amplitude as the drop volume decreases (Fig. 3).
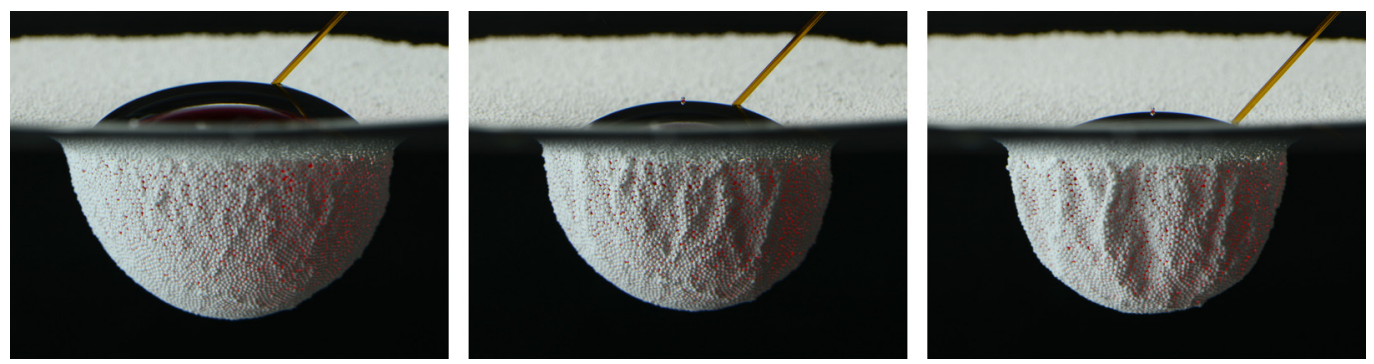

FIG. 3. Liquid is withdrawn from the water drop. Its volume decreases from left to right: $V=1.8,1.2$, and 1.0 mL. DOI: http://dx.doi.org/10.1103/APS.DFD.2015.GFM.P0008

[1] Y. Amarouchene, G. Cristobal, and H. Kellay, Noncoalescing Drops, Phys. Rev. Lett. 87, 206104 (2001).

[2] Y. Couder, E. Fort, C.-H. Gautier, and A. Boudaoud, From Bouncing to Floating: Noncoalescence of Drops on a Fluid Bath, Phys. Rev. Lett. 94, 177801 (2005).

[3] D. E. Tambe and M. M. Sharma, The effect of colloidal particles on fluid-fluid interfacial properties and emulsion stability, Adv. Colloid Interface Sci. 52, 1 (1994).

[4] M. Abkarian, S. Protière, J. M. Aristoff, and H. A. Stone, Gravity-induced encapsulation of liquids by destabilization of granular rafts, Nat. Commun. 4, 1895 (2013).

[5] T. S. Horozov and B. P. Binks, Particle-stabilized emulsions: A bilayer or a bridging monolayer? Angew. Chem. Int. Ed. 45, 773 (2006). 